14

\title{
Применение метода диэлектрической спектроскопии для исследования свойств сыворотки крови мышей со злокачественным асцитом
}

\author{
(C) Ж.А. Сальникова ${ }^{1}$, А.П. Смирнов ${ }^{1}$, А.А. Богданов ${ }^{2,3}$, Н.А. Верлов ${ }^{2,3}$, Р.А. Кастро ${ }^{1}$ \\ ${ }^{1}$ Российский государственный педагогический университет им. А. И. Герцена, \\ 191186 Санкт-Петербург, Россия \\ ${ }^{2}$ Санкт-Петербургский клинический научно-практический центр специализированных видов \\ медицинской помощи (онкологический), \\ 197758 Санкт-Петербург, Россия \\ ${ }^{3}$ Государственный научно-исследовательский институт особо чистых биопрепаратов \\ Федерального медико-биологического агентства, \\ 197110 Санкт-Петербург, Россия \\ E-mail: recastro@mail.ru
}

Поступило в Редакцию 14 июля 2021 г.

В окончательной редакции 26 сентября 2021 г.

Принято к публикации 30 сентября 2021 г.

Приведены результаты исследования методом диэлектрической спектроскопии высокочастотных релаксационных процессов в сыворотке крови лабораторных животных (мышей): интактных (здоровых) и привитых асцитной формой карциномы Эрлиха. С использованием формализма электрического модуля проведен расчет релаксационных параметров для образцов сыворотки крови двух исследуемых систем.

Ключевые слова: электрический модуль, релаксационные параметры, злокачественный асцит.

DOI: $10.21883 / J T F .2022 .01 .51864 .216-21$

\section{Введение}

Диэлектрическая спектроскопия является одним из наиболее информативных физических методов исследования релаксационных свойств диэлектрических сред в широком диапазоне частот $f=10^{-3}-10^{10} \mathrm{~Hz}[1-5]$. В последние годы этот метод широко используется для исследования крови и ее компонент [6-9]. В работе [9] представлены значения $\varepsilon^{\prime}(f)$ и $\varepsilon^{\prime \prime}(f)$ для цельной крови и плазмы для нескольких онкологических больных, измеренные в микроволновом диапазоне. При этом $\varepsilon^{\prime}(f)=80-90 ; \varepsilon^{\prime \prime}(f)=70-15$.

Поскольку сыворотка крови (СК) обладает повышенной электропроводностью, скрывающей релаксационные явления, для их обнаружения мы использовали понятие комплексного электрического модуля - величины, обратной комплексной диэлектрической проницаемости. Опыт показывает, что использование этого метода позволяет выявить релаксационные пики СК, а математический аппарат - определить величины основных параметров релаксации. Данным методом можно пользоваться для любых образцов СК. При этом представляет интерес сравнение релаксационных параметров образцов СК, полученных от здоровых и имеющих патологию биологических объектов. Можно предположить, что параметры СК будут отличаться друг от друга, поскольку при выраженных патологиях изменяется качественный и количественный состав СК, что изменяет характер межмолекулярных взаимодействий между ее элемента- ми и, таким образом, изменяет численные значения релаксационных параметров СК. Идея получать информацию о наличии и развитии патологического процесса в результате исследования температурно-частотных зависимостей релаксационных параметров СК является привлекательной.

Целью настоящей работы было выявление особенностей процессов высокочастотной диэлектрической релаксации и определение релаксационных параметров СК интактных мышей и мышей с асцитной формой карциномы Эрлиха.

\section{1. Теория}

\section{1. Релаксационные уравнения}

В рамках метода диэлектрической спектроскопии для анализа релаксационных свойств диэлектриков используется понятие комплексной диэлектрической проницаемости $\varepsilon^{*}(f)$, которая определяется выражением: $\varepsilon^{*}(f)=\varepsilon^{\prime}(f)-i \varepsilon^{\prime \prime}(f)$, где $f$ - частота приложенного электрического поля, $i$ - мнимая единица, $\varepsilon^{\prime}(f)-$ вещественная диэлектрическая проницаемость среды, характеризующая степень экранировки внешнего электрического поля, $\varepsilon^{\prime \prime}(f)$ - мнимая ее часть, характеризующая поглощение энергии с переводом ее в тепловую форму, т.е. диэлектрические потери. Значения $\varepsilon^{\prime}(f)$ и $\varepsilon^{\prime \prime}(f)$ определяются экспериментально. 
Релаксационные явления в общем виде описываются уравнением Гавриляка-Негами $(\Gamma-\mathrm{H})[10]$ :

$$
\varepsilon^{*}(\omega)=\varepsilon_{\infty}+\frac{\varepsilon_{s}-\varepsilon_{\infty}}{\left(1+\left(i \omega \tau_{0}\right)^{1-\alpha}\right)^{\beta}},
$$

где $\varepsilon_{s}, \varepsilon_{\infty}-$ статическая и высокочастотная диэлектрическая проницаемость $\varepsilon^{\prime}(f)\left(\varepsilon_{s}-\right.$ при $f \rightarrow 0, \varepsilon_{\infty}-$ при $f \rightarrow \infty), \omega=2 \pi f-$ циклическая частота, $\tau_{0}-$ наиболее вероятное время релаксации электрического отклика молекулярных агрегатов или молекул образца, $\alpha$ - ширина спектра времен релаксации, $\beta$ - асимметрия этого спектра. Данные параметры изменяются в следующих пределах: $0 \leq \alpha<1,0<\beta \leq 1$. При этом чем больше величина $\alpha$, тем больше частотная дисперсия численных значений времен релаксации молекул образца $\tau$, т. е. шире релаксационный спектр; чем меньше $\beta$, тем больше степень его асимметрии.

Аналитическое выражение для функции распределения времен релаксаторов $G(\tau)$ относительно $\tau_{0}$ может быть представлено в виде [11]:

$$
G(\tau)=\frac{\left(\frac{\tau}{\tau_{0}}\right)^{\beta(1-\alpha)} \sin (\beta \theta)}{\pi \tau\left[\left(\frac{\tau}{\tau_{0}}\right)^{2(1-\alpha)}+2\left(\frac{\tau}{\tau_{0}}\right)^{(1-\alpha)} \cos (\pi(1-\alpha))+1\right]^{\beta / 2}},
$$

где

$$
\theta=\operatorname{arctg}\left[\frac{\sin (\pi(1-\alpha))}{\left(\frac{\tau}{\tau_{0}}\right)^{(1-\alpha)}+\cos (\pi(1-\alpha))}\right] \quad \text { и } \quad 0 \leq \theta \leq \pi .
$$

При $\alpha=0$ и $\beta=1$ уравнение $\Gamma-\mathrm{H}$ переходит в уравнение Дебая [12]. При этом $G(\tau)$ является дельтафункцией $\delta\left(\tau_{0}\right)$, что соответствует состоянию, когда все молекулы системы обладают одинаковым временем релаксации $\tau_{0}$.

При $\alpha \neq 0$ и $\beta=1$ уравнение $\Gamma-\mathrm{H}$ переходит в уравнение Коула-Коула [13]:

$$
\varepsilon^{*}(\omega)=\varepsilon_{\infty}+\frac{\varepsilon_{s}-\varepsilon_{\infty}}{1+\left(i \omega \tau_{0}\right)^{1-\alpha}} .
$$

При этом $G(\tau)$ является симметричной функцией относительно $\tau_{0}$.

При $\alpha=0$ и $\beta \neq 1$ уравнение $\Gamma-\mathrm{H}$ переходит в уравнение Дэвидсона-Коула [14]:

$$
\varepsilon^{*}(\omega)=\varepsilon_{\infty}+\frac{\varepsilon_{s}-\varepsilon_{\infty}}{\left(1+\omega \tau_{0}\right)^{\beta}}
$$

При этом $G(\tau)$ является асимметричной функцией относительно $\tau_{0}$.

Параметры $\alpha, \beta, \tau \quad$ и $G(\tau)$ являются основными релаксационными параметрами объекта исследования. Их определяют, если имеются релаксационные пики на измеренной экспериментально зависимости $\varepsilon^{\prime \prime}(f)$. Если же этих пиков не удается обнаружить, то и определение данные параметров этим методом становится невозможным. В настоящей работе для их определения мы использовали метод комплексного электрического модуля. Ранее мы уже использовали этот метод для СК при хроническом лимфолейкозе [15].

\section{2. Метод комплексного электрического модуля}

Комплексным электрическим модулем $M^{*}(\omega)$ называется величина обратной комплексной диэлектрической проницаемости, определяемая выражением [16]: $M^{*}(\omega)=M^{\prime}(\omega)+i M^{\prime \prime}(\omega)$. Величины $M^{\prime}(\omega), M^{\prime \prime}(\omega)$ называются соответственно вещественной и мнимой компонентами комплексного электрического модуля. Величина $M^{\prime \prime}(\omega)$ равна

$$
M^{\prime \prime}(\omega)=\frac{\varepsilon^{\prime \prime}(\omega)}{\varepsilon^{\prime 2}(\omega)+\varepsilon^{\prime \prime 2}(\omega)} .
$$

Из уравнения Г-H можно вывести уравнение $M^{\prime \prime}(\omega)[17]$ :

$$
M^{\prime \prime}(\omega)=\frac{M_{\infty} M_{s} A^{\beta}\left(M_{\infty}-M_{s}\right) \sin \beta \phi}{A^{2 \beta} M_{s}^{2}+2 A^{\beta}\left(M_{\infty}-M_{s}\right) M_{s} \cos \beta \phi+\left(M_{\infty}-M_{s}\right)^{2}},
$$

где

$$
\begin{gathered}
M_{\infty}=\frac{1}{\varepsilon_{\infty}}, \quad M_{s}=\frac{1}{\varepsilon_{s}} \\
A=\left[1+2\left(\omega \tau_{0}\right)^{1-\alpha} \sin \frac{\pi \alpha}{2}+\left(\omega \tau_{0}\right)^{2(1-\alpha)}\right]^{1 / 2} \\
\phi=\operatorname{arctg}\left[\frac{\left(\omega \tau_{0}\right)^{1-\alpha} \cos \frac{\pi \alpha}{2}}{1+\left(\omega \tau_{0}\right)^{1-\alpha} \sin \frac{\pi \alpha}{2}}\right] .
\end{gathered}
$$

При этом параметры $\alpha, \beta, \tau_{0}$ имеют тот же физический смысл, что в уравнении (1).

\section{2. Методика эксперимента}

В эксперименте использовали мышей линии CD-1 (самки, масса 22-24 g), полученные из питомника „СТЕ3АР“. Животные содержались в клетках по 5 особей в каждой клетке со свободным доступом к питью и пище (комбинированный корм „Лабораторкорм“б), зоогигиенические показатели у животных в момент начала эксперимента находились в пределах нормы. В рамках исследования были сформированы следующие группы: группа интактных животных (10 особей) и экспериментальная группа (5 особей, которым выполнялось внутрибрюшинное введение суспензии клеток асцитной формы карциномы Эрлиха с концентрацией $3 \cdot 10^{5}$ клеток на мышь). Забор крови у мышей в экспериментальной группе проводился на пятые сутки после введения суспензии. Все животные перед процедурой взятия биологического материала вводились в состояние наркоза (инъекция золетила $100^{\circledR} 2 \mathrm{mg} / \mathrm{kg}$ внутримышечно). Взятые образцы крови помещали в вакуумные пробирки VACUETTE объемом $2.0 \mathrm{ml} \mathrm{c} \mathrm{активатором}$ 
свертывания крови, после чего образцы центрифугировали в течение $10 \mathrm{~min}$ при $2000 \mathrm{rpm}$, далее выполнялся отбор сыворотки. Измерения параметров полученных образцов проводились в день взятия без заморозки. Bce применимые международные, национальные и/или институциональные принципы ухода и использования животных были соблюдены.

Измерения диэлектрических спектров образцов СК были выполнены на спектрометре „Concept-81“ (Novocontrol Technologies $\mathrm{GmbH}$ ), который предназначен для исследования диэлектрических и электропроводящих свойств широкого класса материалов, в том числе жидкостей [18]. Объем измерительной ячейки составлял $60 \mu 1$. Измерения производились в диапазоне частот $f=10^{6}-1.5 \cdot 10^{9} \mathrm{~Hz}$ при комнатной температуре $\left(T=20^{\circ} \mathrm{C}\right)$.

\section{3. Результаты и обсуждение}

Измеряли частотные зависимости вещественной $\varepsilon^{\prime}(f)$ и мнимой $\varepsilon^{\prime \prime}(f)$ частей диэлектрической проницаемости для образцов СК, полученных от интактных животных и животных с асцитной формой карциномы Эрлиха. По формуле (5) строили частотную зависимость электрического модуля $M^{\prime \prime}(\omega)$ для каждого животного. Результаты для образцов СК от интактных животных представлены на рис. 1, для образцов СК от животных, привитых карциномой Эрлиха, - на рис. 2. Экспериментальные данные были аппроксимированы кривой по формуле (6). При этом были получены релаксационные параметры $\alpha, \beta, \tau_{0}$, которые определяли эмпирически на основе принципа наилучшей аппроксимации экспериментальных результатов теоретической кривой $M^{\prime \prime}(\omega)$. Результаты аппроксимации представлены в таблице. Для образца I (рис. 2) представлены результаты аппроксимации только высокочастотного (правого) релаксационного пика. Для всех образцов СК от интактных животных при $T=20^{\circ} \mathrm{C}$ параметр $\alpha$ оказался равным $0(\alpha=0)$, а параметр $\beta \neq 1$, следовательно,

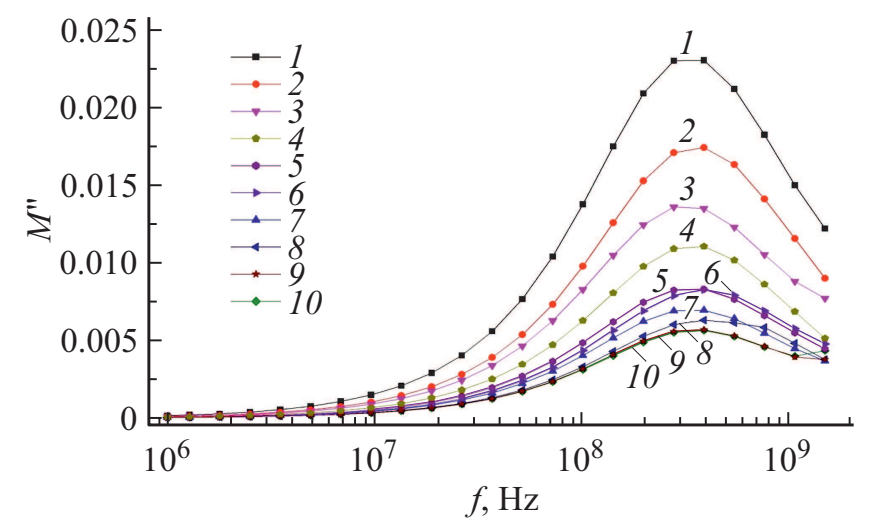

Рис. 1. Частотная зависимость мнимой части электрического модуля $M^{\prime \prime}(f)$ образцов СК, полученных от интактных животных: 1-10 - номер животного (см. таблицу).

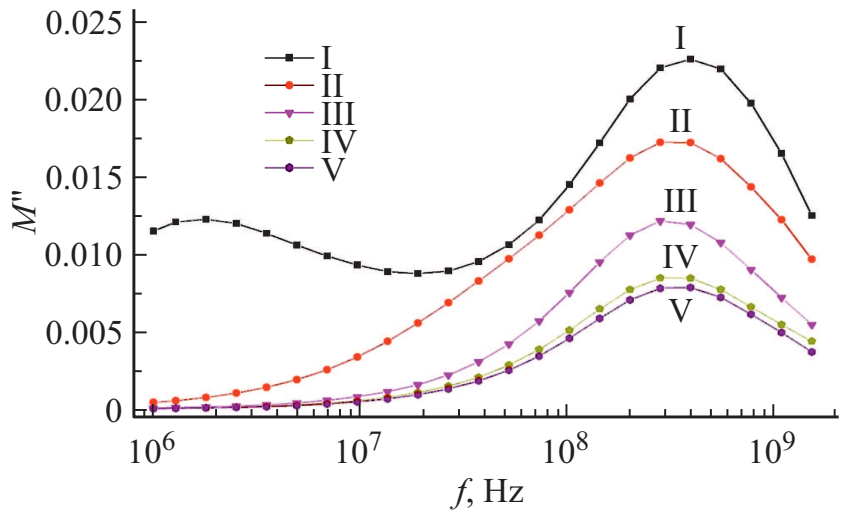

Рис. 2. Частотная зависимость мнимой части электрического модуля $M^{\prime \prime}(f)$ образцов СК, полученных от животных, привитых асцитной формой карциномы Эрлиха: I-V - номер животного (см. таблицу).

Релаксационные параметры $\alpha, \beta, \tau_{0}$ для образцов СК, полученных от интактных и привитых асцитной формой карциномы Эрлиха животных

\begin{tabular}{|c|c|c|c|}
\hline Номер образца* & $\alpha$ & $\beta$ & $\tau_{0}(c)$ \\
\hline 1 & 0.00 & 0.85 & $1.03 \cdot 10^{-9}$ \\
\hline 2 & 0.00 & 0.91 & $1.38 \cdot 10^{-9}$ \\
\hline 3 & 0.00 & 0.85 & $8.42 \cdot 10^{-10}$ \\
\hline 4 & 0.00 & 0.80 & $8.30 \cdot 10^{-10}$ \\
\hline 5 & 0.00 & 0.67 & $4.52 \cdot 10^{-10}$ \\
\hline 6 & 0.00 & 0.56 & $4.44 \cdot 10^{-10}$ \\
\hline 7 & 0.00 & 0.62 & $6.12 \cdot 10^{-10}$ \\
\hline 8 & 0.00 & 0.78 & $5.34 \cdot 10^{-10}$ \\
\hline 9 & 0.00 & 0.66 & $5.32 \cdot 10^{-10}$ \\
\hline 10 & 0.00 & 0.91 & $1.05 \cdot 10^{-9}$ \\
\hline I & 0.26 & 1.00 & $1.82 \cdot 10^{-9}$ \\
\hline II & 0.25 & 1.00 & $5.95 \cdot 10^{-10}$ \\
\hline III & 0.01 & 0.90 & $5.72 \cdot 10^{-10}$ \\
\hline IV & 0.01 & 0.82 & $6.01 \cdot 10^{-10}$ \\
\hline V & 0.01 & 0.98 & $2.29 \cdot 10^{-8}$ \\
\hline
\end{tabular}

Примечание. * 1-10 - интактные животные, I-V - животные, привитые асцитной формой карциномы Эрлиха.

релаксационный спектр для них описывается уравнением Дэвидсона-Коула (4). Этот результат показывает, что молекулы СК обладают различающимися друг от друга временами релаксации $\tau$, расположенными на горизонтальной шкале несимметрично относительно $\tau_{0}$, т. е. функция $G(\tau)$ является асимметричной.

На рис. 3 представлена функция $G(\tau)$, полученная по результатам исследования СК от животных 1 и 3 . Для 

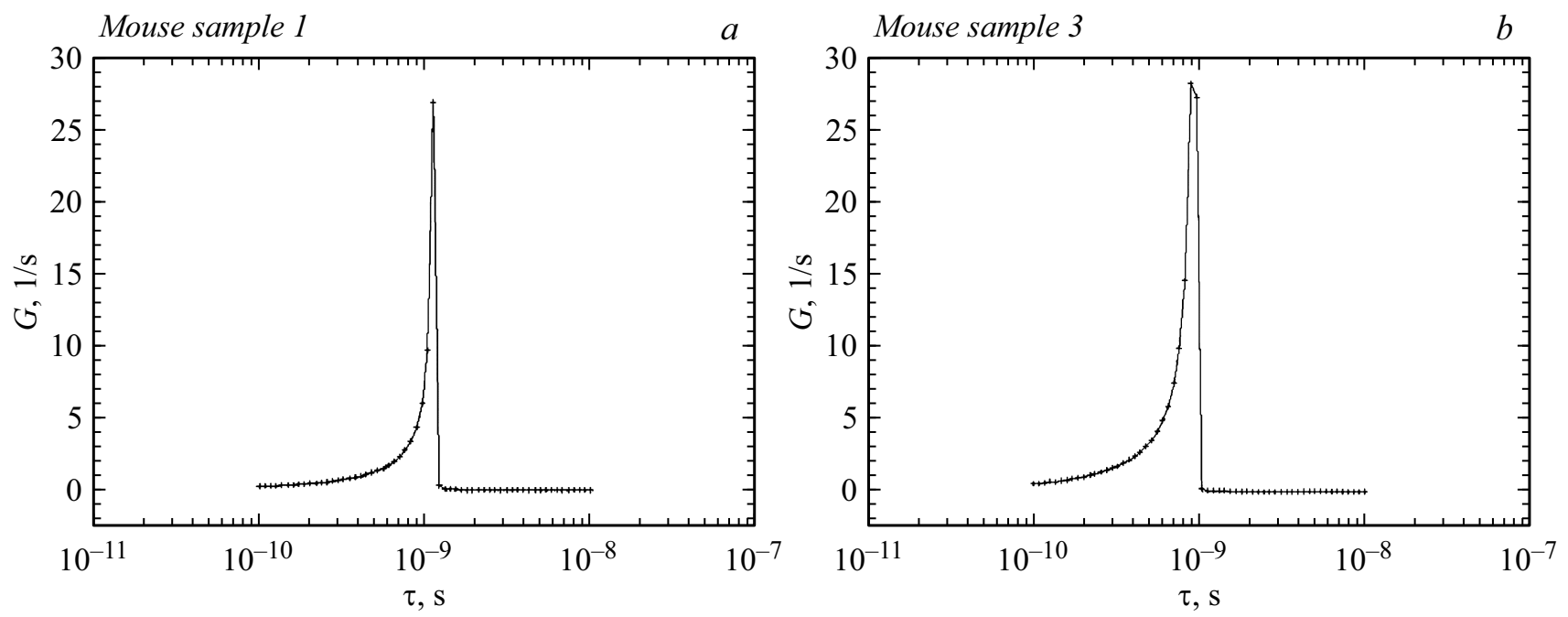

Рис. 3. Функция распределения времен релаксации $G(\tau)$ для интактных животных: $a$ - животное $1, b-$ животное 3 (см. таблицу).
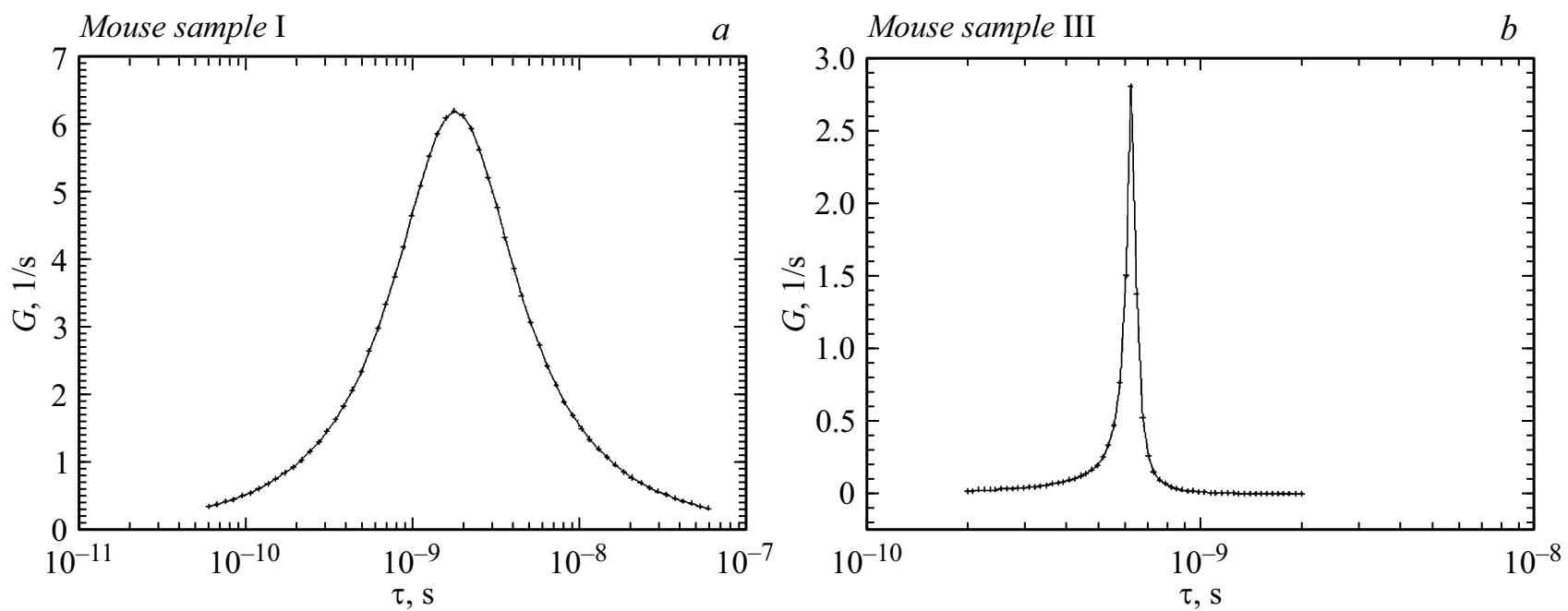

Рис. 4. Функция распределения времен релаксации $G(\tau)$ для животных, привитых асцитной формой карциномы Эрлиха: $a-$ животное I, $b$ - животное III (см. таблицу).

образцов СК от всех остальных интактных животных данные графики выглядят аналогично: распределение релаксаторов по временам релаксации асимметрично относительно $\tau_{0}$.

Как видно из таблицы, для образцов СК от животных, привитых асцитной формой карциномы Эрлиха, при $T=20^{\circ} \mathrm{C}$ параметр $\alpha \neq 0$. Для образцов I и II $\beta=1$, откуда следует, что релаксационный спектр для них описывается уравнением Коула-Коула (3). Следствием данного уравнения является то, что молекулы обладают различными временами релаксации $\tau$, расположенными симметрично относительно $\tau_{0}$, т.е. функция $G(\tau)$ является симметричной. На рис. 4 представлена функция $G(\tau)$ для животных I и III. Как видно из графиков, распределение симметрично. Визуальное отличие рис. 4, $a$ от рис. $4, b$ связано с большей величиной параметра $\alpha$ для образца I. Для образца II функция $G(\tau)$ выглядит так же, как для образца I, для образцов животных IV, V функция $G(\tau)$ такая же, как и для образца III. Для образцов III, IV, V $\alpha \neq 0$ и $\beta \neq 1$, однако для них среднее значение параметра $\beta(\langle\beta\rangle=0.90)$ значительно ближе к единице, чем для образцов интактных животных $(\langle\beta\rangle=0.76)$. Следовательно, их релаксационный спектр можно приближенно описывать уравнением Коула-Коула (3), как и для образцов СК I и II.

Наличие в образцах сыворотки макромолекул с различными временами релаксации $\tau$ следует из теоретического описания диэлектрической релаксации для независимых диполей с несколькими дискретными состояниями ориентации [19]. Учет кооперативного характера переориентации макромолекулы, при котором переориентация диполя осуществляется в виде одиночных 
переходов, но вероятность переориентации или энергия активации переориентации зависит от ориентации соседей [20], приводит к возникновению спектра времен релаксации $\tau$, показанного на рис. 3 и 4. Для более вытянутых молекул спектр времен релаксации будет асимметричным (поскольку их перескоки из одного положения равновесия в другое зависят от ориентации соседей и энергии взаимодействия с ними), а для макромолекул, близких по форме к шарообразным, спектр времен будет симметричным (поскольку для шарообразных молекул ориентация соседей неважна). Таким образом, отличие в релаксационных спектрах образцов СК от интактных животных и животных, привитых карциномой Эрлиха, возможно, связано с изменением конформации макромолекул белка в составе сыворотки крови при развитии злокачественного процесса.

\section{Заключение}

Использование метода комплексного электрического модуля позволяет определять релаксационные параметры $\alpha, \beta, \tau$ и функцию $G(\tau)$ для образцов СК, полученных от интактных и привитых асцитной формой карциномы Эрлиха животных. При температуре $20^{\circ} \mathrm{C}$ для интактных и больных животных данные параметры несколько отличаются друг от друга. Эти различия, вероятно, могут быть более значимыми при физиологических температурах $35-41^{\circ} \mathrm{C}$. При развитии заболеваний может изменяться состав СК, что влияет на межмолекулярные взаимодействия, следовательно, и на релаксационные свойства СК. Установление взаимосвязи изменения релаксационных параметров с наличием и стадией заболевания, например, онкологического, может быть темой дальнейших исследований. Изучение вопроса возможного изменения конформации молекул белка в составе СК при развитии патологического процесса требует применения дополнительных физических методов исследования. При этом принципиально необходимо выполнение корреляционного анализа между данными, получаемыми методом диэлектрической спектроскопии, и данными биохимических анализов крови для различных заболеваний.

Полученные результаты позволяют предположить, что метод диэлектрической спектроскопии в перспективе может быть использован для диагностики заболеваний, например онкологических, основанной на выявлении особенностей молекулярных физических процессов жидкостей организма.

\section{Конфликт интересов}

Авторы заявляют, что у них нет конфликта интересов.

\section{Список литературы}

[1] Б.И. Сажин. Электрические свойства полимеров (Химия, Л., 1986)

[2] Э.Р. Блайт, Д. Блур. Электрические свойства полимеров (Физматлит, М., 2008)

[3] Ю.А. Гороховатский, Е.А. Карулина, Д.Э. Темнов. Физика полимерных диэлектриков (Изд-во РГПУ им. А.И. Герцена, СПб., 2013)

[4] R.A. Castro, A.I. Ignatiev, N.V. Nikonorov, A.I. Sidorov, M.V. Stolyarchuk. J. Non-Crystall. Solid., 461, 72 (2017).

[5] N.A. Nikonorova, A.A. Kononov, H.T. Dao, R.A. Castro. J. Non-Crystall. Solid., 511, 109 (2019).

[6] M. Wolf, R. Gulich, P. Lunkenheimer, A. Loidl. Biochim. Biophys. Acta, 1810 (8), 727 (2011).

[7] K. Asami. J. Non-Crystall. Solid., 305, 268 (2002).

[8] T. Chelidze. J. Non-Crystall. Solid., 305, 285 (2002).

[9] А.Н. Романов, Е.Ю. Винокурова, А.О. Ковригин, А.Ф. Лазарев, В.А. Лубенников, Н.А. Романова, С.А. Комаров. Диэлектрические характеристики биологических жсикостей человека при развитии онкологических заболеваний (микроволновый диапазон) (Азбука, Барнаул, 2008)

[10] S. Havriliak, S. Negami. J. Polymer Science Part C, 14 (1), 99 (1966).

[11] S. Havriliak, S. Negami. Polymer, 8, 161 (1967).

[12] П. Дебай Полярные молекулы (ГНТИ, М.-Л., 1931)

[13] K.S. Cole, R.H. Cole. J. Chem. Phys., 9, 341 (1941)

[14] D.W. Davidson, R.H. Cole. J. Chem. Phys., 19, 1484 (1951); 18, 1417 (1950)

[15] Zh.A. Salnikova, L.V. Plotnikova, A.P. Smirnov, A.D. Garifullin, A.Yu. Kuvshinov, S.V. Voloshin. A.M. Polyanichko. AIP Conf. Proceed. 2308, 030018 (2020); DOI: $10.1063 / 5.0035270$

[16] N.G. McCrum, B.E. Read, G. Williams In: Anealastic and Dielectric Effecrts in Polymeric Solids (Willey, London, 1967), p. 108-111.

[17] Zh.A. Salnikova, A.A. Kononov. AIP Conf. Proceed. 2308, 030017 (2020); DOI: 10.1063/5.0034028

[18] Электронный ресурс. Описание прибора „Novocontrol Concept-81“ https://ckpo.herzen.spb.ru/?page=dielectrici

[19] Г. Фрелих. Теория диэлектриков (ИИЛ, М., 1960)

[20] Ю.Я. Готлиб, А.А. Даринский, Ю.Е. Светлов. Физическая кинетика макромолекул (Химия, Л., 1986) 